\title{
A review: modified agricultural by-products for the development and fortification of food products and nutraceuticals
}

\begin{abstract}
Producing more food for a growing population in the coming decades, while at the same time combating environmental issues, is a huge challenge faced by the worldwide population. The risks that come with climate change make the mission more daunting. Billion tons of agriculture by-products are produced each year along the agricultural and food processing processes. There is a need to take further actions on exploring the inner potential of agrowaste to stand out as food ingredient to partially or fully substitute the foods in orthodox list. Some of the agro-waste contains the most valuable nutrients in the plant and it is truly a "waste" to dispose any of them. Furthermore, the paper aims at discussing the possible methods of modification to improve the safety and feasibility of the agro-waste either through physical, chemical or microbiological ways. The safety issues and bioactivity contains in the agro-waste also been discussed to present the better overall ideas about the employing of agro-waste in food applications.
\end{abstract}

